For Supplementary Information

\title{
Synthesis and Catalytic Activity of Cationic Allyl Complexes of Ni Stabilized by a Single N-Heterocyclic Carbene Ligand.
}

Juan Cámpora, ${ }^{a}{ }^{a}$ Laura Ortiz de la Tabla, ${ }^{a}$ Pilar Palma, ${ }^{a}$ Eleuterio Álvarez, ${ }^{a}$ Fernando J. $\operatorname{Lahoz}^{b}$ and Kurt Mereiter ${ }^{c}$

${ }^{a}$ Instituto de Investigaciones Químicas, CSIC-Universidad de Sevilla. c/ Americo Vespucio, 49. 41018, Sevilla, Spain. Fax: +34 954460565; Tel: +34 954489555.E-mail: campora@iiq.csic.es

${ }^{b}$ Instituto de Ciencia de Materiales de Aragón, CSIC-Universidad de Zaragoza. Pza. San Francisco S/N, Zaragoza, Spain.

${ }^{b}$ Department of Chemistry, Vienna University of Technology, Getreidemarkt 9/164SC, Vienna, Austria. 


\section{General Information.}

All preparations and other operations were carried out under oxygen-free nitrogen by conventional Schlenk techniques. Solvents were rigorously dried and degassed before use. Microanalyses were performed by the Microanalytical Service of the Instituto de Investigaciones Químicas (Sevilla, Spain). Infrared spectra were recorded on a Bruker Vector 22 spectrometer, and NMR spectra on Bruker DRX 300, 400 and $500 \mathrm{MHz}$ spectrometers. The ${ }^{1} \mathrm{H}$ and ${ }^{13} \mathrm{C}\left\{{ }^{1} \mathrm{H}\right\}$ resonances of the solvent were used as the internal standard but the chemical shifts are reported with respect to TMS. Where necessary, the spectral assignations have been helped with 2-D COSY, HETCOR and NOESY spectra. Mixtures of styrene oligomers were analyzed in an Agilent model 6890 gas chromatograph, with TCD detector or a MS-GC ThermoQuest Automass Multi, both equipped with a Technochroma colum TR-110232 of $30 \mathrm{~m}$ x $0.25 \mathrm{~mm}$. Polymers were analyzed at Centro de Tecnología Repsol-YPF (GPC low temperature and NMR). Compound $\mathbf{1}^{1}$ and the NHC ligands I-DiPP ${ }^{2}$ and I-Me, $\mathrm{Me}^{3}$ were prepared according to published procedures.

\section{Synthesis of $\left[\mathrm{Ni}\left(\eta^{3}-\mathrm{C}_{3} \mathrm{H}_{5}\right)\left(\mathrm{OC}_{6} \mathrm{H}_{3}-2,6-{ }^{\mathrm{i}} \mathrm{Pr}_{2}\right)(\right.$ IDiPP $\left.)\right](2 \mathrm{a})$}

$732 \mathrm{mg}$ of IDiPP (1.88 mmol) dissolved in $30 \mathrm{~mL}$ of $\mathrm{Et}_{2} \mathrm{O}$ was added to a solution of $522 \mathrm{mg}(0.94 \mathrm{mmol})$ compound $1 \mathrm{in} 30 \mathrm{~mL}$ of $\mathrm{Et}_{2} \mathrm{O}$ cooled at $-40{ }^{\circ} \mathrm{C}$. The mixture was allowed to reach room temperature and stirred one hour at this temperature. The solvent was evaporated under vacuum and the residue extracted with $80 \mathrm{~mL}$ of hexane. After filtration and partial concentration of the solution, compound 2a was isolated as red crystals after cooling at $-30{ }^{\circ} \mathrm{C}$ in $52 \%$ yield. ${ }^{1} \mathrm{H}$ NMR $\left(\mathrm{C}_{6} \mathrm{D}_{6}\right): 0.47\left(\mathrm{~d}, 1 \mathrm{H},{ }^{3} J_{\mathrm{HH}}=12\right.$ $\mathrm{Hz}$, anti $\mathrm{CHH}$, allyl), 0.95-1.11 (m, 12H, Me, O-DiPP), 1.20-1.43 (m, 24H, Me, I-DiPP), $1.5\left(\mathrm{bd}, 1 \mathrm{H},{ }^{3} J_{\mathrm{HH}}=6 \mathrm{~Hz}, \operatorname{synCHH}\right.$, allyl $), 2.26\left(\mathrm{~d}, 1 \mathrm{H},{ }^{3} J_{\mathrm{HH}}=14 \mathrm{~Hz}\right.$, antiCHH, allyl $)$, 
$2.44\left(\mathrm{bd}, 1 \mathrm{H},{ }^{3} J_{\mathrm{HH}}=6 \mathrm{~Hz}, \operatorname{synCHH}\right.$, allyl), $2.92\left(\mathrm{~h}, 1 \mathrm{H},{ }^{3} J_{\mathrm{HH}}=7 \mathrm{~Hz}, \mathrm{CH}, \mathrm{O}-\mathrm{DiPP}\right), 3.6$ (h, $\left.1 \mathrm{H},{ }^{3} J_{\mathrm{HH}}=7 \mathrm{~Hz}, \mathrm{CH}, \mathrm{O}-\mathrm{DiPP}\right), 4.87$ (m, 1H, central CH, allyl), 6.37 (s, 2H, $\mathrm{NCHCHN}), 6.86\left(\mathrm{t}, 2 \mathrm{H},{ }^{3} J_{\mathrm{HH}}=7 \mathrm{~Hz}, p\right.$-CHarom, I-DiPP ), 7.05-7.35 (m, 7H, CHarom). ${ }^{13} \mathrm{C}\left\{{ }^{1} \mathrm{H}\right\}$ NMR $\left(\mathrm{C}_{6} \mathrm{D}_{6}\right): 22.7$ (Me IDiPP), 22.8 (Me IDiPP), 23.8 (CH, O-DiPP), 24.2 ( $C H$ or $M e$, I-DiPP), 25.0 (CH, O-DiPP), 25.2 (Me, O-DiPP), 25.7 (Me, IDiPP), 26.0 ( $C \mathrm{H}$ or $M e$, IDiPP), 28.5 (bs, $C H$, IDiPP), 28.7 (bs, $C H$, IDiPP), $37.3\left(\mathrm{CH}_{2}\right.$, allyl), 69.6 $\left(\mathrm{CH}_{2}\right.$, allyl), 108.4 (central $\mathrm{CH}$, allyl), 114.6 ( $p \mathrm{CH}$ arom, IDiPP), 122.3 ( $m C \mathrm{H}$ arom, ODiPP), 122.4 ( $m C H$ arom, O-DiPP), 123.6 (bs, $m-C H$ arom IDiPP), 123.9 (bs, $m-C H$ arom IDiPP), 124.3 (NCHCHN), 129.7 ( $p$ CHarom IDiPP), 135.9 (Cq arom), 137.2 (Cq arom), 137.7 (Cq arom), 145.5 (Cq arom), 145.9 (Cq arom), 170.1 (Cq arom), 185.1 (s, $\mathrm{NCN}$ ). Anal. Calcd for $\mathrm{C}_{42} \mathrm{H}_{58} \mathrm{~N}_{2} \mathrm{NiO}$ C, 75.82; H, 8.73; N, 4.21.Found: C, 75.44; $\mathrm{H}$, 8.87; N, 4.14 .

\section{Synthesis of $\left[\mathrm{Ni}\left(\eta^{3}-\mathrm{C}_{3} \mathrm{H}_{5}\right)\left(\mathrm{OC}_{6} \mathrm{H}_{3}-2,6-{ }^{\mathrm{i}} \mathrm{Pr}_{2}\right)(\mathrm{IMe}, \mathrm{Me})\right](2 \mathrm{~b})$}

A solution of compound $1(238 \mathrm{mg}, 0.43 \mathrm{mmol})$ in $20 \mathrm{~mL}$ of $\mathrm{Et}_{2} \mathrm{O}$ cooled at $-50{ }^{\circ} \mathrm{C}$ was treated with a solution of IMe,Me $(106 \mathrm{mg}, 0.86 \mathrm{mmol})$ in $10 \mathrm{~mL}$ of $\mathrm{Et}_{2} \mathrm{O}$. The mixture was stirred one hour at room temperature. The solvent was evaporated under reduced pressure, the residue extracted with $30 \mathrm{~mL}$ of hexanes and the solution centrifuged. Crystallization from $\left(\mathrm{Me}_{3} \mathrm{Si}\right)_{2} \mathrm{O}$ at $-10^{\circ} \mathrm{C}$ furnished orange crystals of $\mathbf{2 b}$. Yield: 91\%. ${ }^{1} \mathrm{H}$ NMR $\left(\mathrm{C}_{6} \mathrm{D}_{6}\right): 1.20\left(\mathrm{~d}, 1 \mathrm{H},{ }^{3} J_{\mathrm{HH}}=12 \mathrm{~Hz}\right.$, antiCHH, allyl), $1.28(\mathrm{~s}, 6 \mathrm{H}$, $\left.\mathrm{CH}_{3} \mathrm{C}, \mathrm{IMe}, \mathrm{Me}\right), 1.52\left(\mathrm{~d}, 6 \mathrm{H},{ }^{3} J_{\mathrm{HH}}=7 \mathrm{~Hz}, M e, \mathrm{O}-\mathrm{DiPP}\right), 1.59\left(\mathrm{~d}, 6 \mathrm{H},{ }^{3} J_{\mathrm{HH}}=7 \mathrm{~Hz}\right.$, Me,O-DiPP), $1.67\left(\mathrm{bd}, 1 \mathrm{H},{ }^{3} J_{\mathrm{HH}}=6 \mathrm{~Hz}, \operatorname{syn} \mathrm{CHH}\right.$, allyl), $2.53\left(\mathrm{~d}, 1 \mathrm{H},{ }^{3} J_{\mathrm{HH}}=14 \mathrm{~Hz}\right.$, antiCHH, allyl), $2.86\left(\mathrm{bd}, 1 \mathrm{H},{ }^{3} J_{\mathrm{HH}}=7 \mathrm{~Hz}, \operatorname{syn} \mathrm{CHH}\right), 3.55\left(\mathrm{~s}, 6 \mathrm{H}, \mathrm{CH}_{3} \mathrm{~N}, \mathrm{IMe}, \mathrm{Me}\right)$, $4.47\left(\mathrm{~h}, 2 \mathrm{H},{ }^{3} J_{\mathrm{HH}}=7 \mathrm{~Hz}, \mathrm{CH}, \mathrm{O}-\mathrm{DiPP}\right), 5.20\left(\mathrm{~h}, 1 \mathrm{H},{ }^{3} J_{\mathrm{HH}}=7 \mathrm{~Hz}\right.$, central CH, allyl), $6.99\left(\mathrm{t}, 1 \mathrm{H},{ }^{3} J_{\mathrm{HH}}=8 \mathrm{~Hz}, p\right.$-CHarom, O-DiPP), $7.32\left(\mathrm{~d}, 2 \mathrm{H},{ }^{3} J_{\mathrm{HH}}=7 \mathrm{~Hz}, m-\mathrm{CHarom}, \mathrm{O}-\right.$ 
DiPP). ${ }^{13} \mathrm{C}\left\{{ }^{1} \mathrm{H}\right\}$ NMR $\left(\mathrm{C}_{6} \mathrm{D}_{6}\right): 7.9\left(\mathrm{CH}_{3} \mathrm{C}, \mathrm{IMe}, \mathrm{Me}\right), 23.7$ (Me, O-DiPP), 27.4 (CH, ODiPP), $33.7\left(\mathrm{CH}_{3} \mathrm{~N}, \mathrm{IMe}, \mathrm{Me}\right), 36.4\left(\mathrm{CH}_{2}\right.$, allyl), $66.1\left(\mathrm{CH}_{2}\right.$, allyl $), 107.5$ ( $\mathrm{CH}$, allyl), 114.3 ( $p$ CH arom, O-DiPP), 122.3 ( $m$ CH arom, O-DiPP), $124.4\left(\mathrm{CH}_{3} C, \mathrm{IMe}, \mathrm{Me}\right)$, 128.6 ( o Cq arom, O-DiPP), 136.7 (ipso Cq arom, O-DiPP), 179.3 (NCN, IMe,Me). Anal. Calcd for $\mathrm{C}_{22} \mathrm{H}_{34} \mathrm{~N}_{2} \mathrm{NiO}$ : C, 65.86; H, 8.54; N, 6.98.Found: C, 65.80; H, 8.45; N, 6.94.

\section{Synthesis of $\left[\mathrm{Ni}\left(\eta^{3}-\mathrm{C}_{3} \mathrm{H}_{5}\right)(\mathrm{IDiPP})\left(\mathrm{H}_{2} \mathrm{O}\right)\right] \mathrm{BAr}_{4}{ }_{4}(3)$}

$20 \mathrm{~mL}$ of diethyl ether was added to $598 \mathrm{mg}$ of $\mathbf{2 a}(0.9 \mathrm{mmol})$ and $182 \mathrm{mg}$ of $\mathrm{H}\left(\mathrm{Et}_{2} \mathrm{O}\right)_{2} \mathrm{BAr}_{4}^{\prime}(0.9 \mathrm{mmol})$ at $-40{ }^{\circ} \mathrm{C}$. The mixture was stirred 30 minutes at this temperature. The solvent was evaporated and the yellow residue of $\mathbf{3}$ was washed with 2 x $10 \mathrm{~mL}$ of hexanes. Yield: $95 \% .{ }^{1} \mathrm{H}$ NMR $\left(\mathrm{CD}_{2} \mathrm{Cl}_{2},-20{ }^{\circ} \mathrm{C}\right): 0.91\left(\mathrm{~d}, 1 \mathrm{H},{ }^{3} J_{\mathrm{HH}}=12 \mathrm{~Hz}\right.$ $\mathrm{CHH}$ allyl $), 1.14\left(\mathrm{~d}, 6 \mathrm{H},{ }^{3} J_{\mathrm{HH}}=7 \mathrm{~Hz}, M e\right.$, IDiPP), $1.16\left(\mathrm{~d}, 6 \mathrm{H},{ }^{3} J_{\mathrm{HH}}=7 \mathrm{~Hz}, M e\right.$, IDiPP), $1.32\left(\mathrm{~d}, 6 \mathrm{H},{ }^{3} J_{\mathrm{HH}}=6 \mathrm{~Hz}, M e, \mathrm{IDiPP}\right), 1.46\left(\mathrm{~d}, 6 \mathrm{H},{ }^{3} J_{\mathrm{HH}}=7 \mathrm{~Hz}, M e, \mathrm{IDiPP}\right), 2.15(\mathrm{bd}$, $1 \mathrm{H},{ }^{3} J_{\mathrm{HH}} \approx 5 \mathrm{~Hz}$, syn $\mathrm{CH}$ allyl), 2.48 (m, 3H, two overlapping signals, anti $\mathrm{CH}$ allyl and CH IDiPP) 2.75 (h, 2H, ${ }^{3} J_{\mathrm{HH}}=7, \mathrm{CH}$ IDiPP), 3.49 (bm, 1H, syn CHH allyl), 5.14 (m, 1H, central $\mathrm{CH}$, allyl), 7.25 (s, 2H, $\mathrm{NCHCHN}$, IDiPP), 7.41 (m, 4H, CH arom, IDiPP), 7.55 (m, 4H, CH arom, BAr ${ }^{\mathrm{F}}$ ); 7.58 (m, 2H, CHarom, IDiPP), 7.71 (bs, 8H, CHarom, BAr $\left.{ }^{\mathrm{F}}\right) .{ }^{13} \mathrm{C}\left\{{ }^{1} \mathrm{H}\right\}$ NMR $\left(\mathrm{CD}_{2} \mathrm{Cl}_{2},-20{ }^{\circ} \mathrm{C}\right): 23.1$ (Me, IDiPP), 23.4 (s, (Me, IDiPP), 25.7 (Me, IDiPP), 26.4 (Me, IDiPP), 29.1 ( $C H$, IDiPP), 29.3 ( $C H$, IDiPP), 43.2 (bs, $\mathrm{CH}_{2}$, allyl), 69.9 (bs, $\mathrm{CH}_{2}$, allyl), $114.9\left(\mathrm{CH}\right.$, allyl), 117.9 (s, $\mathrm{CH}$ arom $\left.\mathrm{BAr}^{\mathrm{F}}\right), 124.9$ $\left(\mathrm{q},{ }^{1} J_{\mathrm{CF}}=272 \mathrm{~Hz}, C_{\mathrm{F}}, \mathrm{BAr}^{\mathrm{F}}\right), 125.3(\mathrm{~s}, \mathrm{NCHCHN}), 125.5$ (s, CH arom IDiPP), 125.6 (CH arom IDiPP), 129.1 (q, ${ }^{2} J_{\mathrm{CF}}=32 \mathrm{~Hz}, C \mathrm{H}$ arom $\left.\mathrm{BAr}{ }^{\mathrm{F}}\right), 131.6(C \mathrm{H}$ arom IDiPP), $145.1\left(C_{\mathrm{q}}\right.$ arom IDiPP), $145.5(C \mathrm{q}$ arom IDiPP), 145.8 (Cq arom IDiPP), $162.2(\mathrm{q}$ $\left.1: 1: 1: 1,{ }^{l} \mathrm{~J}_{\mathrm{CB}}=50 \mathrm{~Hz}, \mathrm{BAr}{ }^{\mathrm{F}}\right), 176.4(\mathrm{NCN})$. IR (nujol): $3636 \mathrm{w} \mathrm{cm}^{-1}\left(v(\mathrm{O}-\mathrm{H}), 3506 \mathrm{~m} \mathrm{~cm}^{-}\right.$ ${ }^{1}\left(v(\mathrm{O}-\mathrm{H}), 1611 \mathrm{~cm}^{-1}(\delta(\mathrm{H}-\mathrm{O}-\mathrm{H})\right.$ and $v(\mathrm{C}=\mathrm{N}))$. Anal. Calcd for $\mathrm{C}_{64} \mathrm{H}_{55} \mathrm{BF}_{24} \mathrm{~N}_{2} \mathrm{NiO}: \mathrm{C}$, 
54.37; H, 4.05; N, 2.05. Found: C, 54.19; $\mathrm{H}, 4.12 ; \mathrm{N}, 1.82 \mathrm{MS}(\mathrm{ESI})$ for $\mathrm{C}_{30} \mathrm{H}_{41} \mathrm{~N}_{2} \mathrm{Ni}^{+}$: $\mathrm{m} / \mathrm{z} 487\left(\mathrm{M}^{+}-\mathrm{H}_{2} \mathrm{O}\right), \mathrm{m} / \mathrm{z} 389\left(\mathrm{IDiPP}^{+}\right)$.

\section{Synthesis of $\left[\mathrm{Ni}\left(\eta^{3}-\mathrm{C}_{3} \mathrm{H}_{5}\right)(\mathrm{IDiPP})\left(\mathrm{NCCH}_{3}\right)\right] \mathrm{BAr}_{4}{ }_{4}(4 \mathrm{a})$}

Method A: A solution of $\mathrm{H}\left(\mathrm{Et}_{2} \mathrm{O}\right)_{2} \mathrm{BAr}_{4}{ }_{4}(202 \mathrm{mg}, 0.2 \mathrm{mmol})$ in $5 \mathrm{~mL}$ of $\mathrm{CH}_{3} \mathrm{CN}$ was added to a solution of $\mathbf{2 a}(133 \mathrm{mg}, 0.2 \mathrm{mmol})$ in $30 \mathrm{~mL}$ of the same solvent cooled at $-40{ }^{\circ} \mathrm{C}$. The mixture was stirred one hour at room temperature and the suspension filtrated. The yellow residue of $\mathbf{4 a}$ was washed with $10 \mathrm{~mL}$ of hexanes. Compound $\mathbf{4 a}$ can be crystallized from $\mathrm{CH}_{3} \mathrm{CN}$ affording yellow crystals. Yield: 50\%. Method B: 98 $\mathrm{mg}(0.06 \mathrm{mmol})$ of $\left[\mathrm{Ni}\left(\eta^{3}-\mathrm{C}_{3} \mathrm{H}_{5}\right)(\mathrm{IDiPP})\left(\mathrm{OH}_{2}\right)\right] \mathrm{BAr}_{4}{ }_{4}, \mathbf{3}$, were dissolved in $10 \mathrm{~mL}$ of $\mathrm{CH}_{3} \mathrm{CN}$ and stirred 30 minutes at room temperature. The solvent was evaporated under vacuum, and the residue recrystallized from $\mathrm{CH}_{3} \mathrm{CN}$ at $-30{ }^{\circ} \mathrm{C}$. Compound $4 \mathrm{a}$ was isolated as yellow crystals in $86 \%$ yield. ${ }^{1} \mathrm{H} \mathrm{NMR}\left(\mathrm{CD}_{2} \mathrm{Cl}_{2}, 25^{\circ} \mathrm{C}\right.$ ): 1.18 (two overlapping doublets, ${ }^{3} J_{\mathrm{HH}} \approx 7 \mathrm{~Hz}, 12 \mathrm{H}, M e$, IDiPP), 1.32 (obscured by a Me signal, $1 \mathrm{H}$, anti $\mathrm{CHH}$, allyl), 1.33 (bd, $6 \mathrm{H},{ }^{3} J_{\mathrm{HH}}=7 \mathrm{~Hz}, M e$, IDiPP), $1.40\left(\mathrm{bd}, 6 \mathrm{H},{ }^{3} J_{\mathrm{HH}}=7 \mathrm{~Hz}\right.$, bd, $6 \mathrm{H},{ }^{3} J_{\mathrm{HH}}=7 \mathrm{~Hz}, M e$, IDiPP), 1.98 (s, 3H, CH $\mathrm{CHN}_{3}$ ), 2.50 (bm, 2H, CH DiPP), 2.50 (obscured by a $\mathrm{CH}$ signal, $1 \mathrm{H}$, anti $\mathrm{CH}$, allyl), 2.69 (bd $1 \mathrm{H},{ }^{3} J_{\mathrm{HH}} \approx 7 \mathrm{~Hz}$, syn $\mathrm{CH}$, allyl); $2.79\left(\mathrm{~h},{ }^{3} J_{\mathrm{HH}} \approx 7 \mathrm{~Hz}, 2 \mathrm{H}, \mathrm{CH} \mathrm{IDiPP}\right), 3.61\left(\mathrm{bd}, 1 \mathrm{H},{ }^{3} J_{\mathrm{HH}}=7 \mathrm{~Hz}\right.$, syn $\mathrm{CHH}$, allyl), $4.97\left(\mathrm{~m}, 1 \mathrm{H}\right.$, central $\mathrm{CH}$, allyl), $7.28(\mathrm{~s}, 2 \mathrm{H}, \mathrm{NCHCHN}), 7.39\left(\mathrm{t}, 4 \mathrm{H},{ }^{3} J_{\mathrm{HH}}=8 \mathrm{~Hz}, p \mathrm{CH}\right.$ arom IDiPP), $7.50\left(\mathrm{bs}, 4 \mathrm{H}, \mathrm{CH}\right.$ arom $\left.\mathrm{BAr}^{\mathrm{F}}\right), 7.53\left(\mathrm{~d}, 1 \mathrm{H},{ }^{3} J_{\mathrm{HH}}=8 \mathrm{~Hz}, m \mathrm{CH}\right.$ arom IDiPP), 7.70 (bs, 8H CH arom BAr $\left.{ }^{\mathrm{F}}\right) .{ }^{13} \mathrm{C}\left\{{ }^{1} \mathrm{H}\right\} \mathrm{NMR}\left(\mathrm{CD}_{2} \mathrm{Cl}_{2}, 25^{\circ} \mathrm{C}\right): 3.9\left(\mathrm{CH}_{3} \mathrm{CN}\right)$, 22.9 (Me, IDiPP), 25.8 (bs, Me, IDiPP), 26.0 (bs, Me, IDiPP), 29.1 (CH, IDiPP), 51.9 ( $\mathrm{CH}_{2}$ allyl), $72.9\left(\mathrm{CH}_{2}\right.$ allyl $), 114.6(\mathrm{CH}$ allyl $), 117.8\left(\mathrm{CH}\right.$ aorm $\left.\mathrm{BAr}^{\mathrm{F}}\right), 124.4(\mathrm{~s}$, $\left.\mathrm{CH}_{3} \mathrm{CN}\right), 124.8\left(m C \mathrm{H}\right.$ arom IDiPP), $125.0\left(\mathrm{q},{ }^{1} J_{\mathrm{CF}}=272 \mathrm{~Hz}, \mathrm{CF}_{3}, \mathrm{BAr}^{\mathrm{F}}\right), 125.9(\mathrm{~s}$, $\mathrm{NCHCHN}), 129.3\left(\mathrm{q}^{2}{ }^{2} J_{\mathrm{CF}}=31 \mathrm{~Hz}, C \mathrm{H}\right.$ arom $\left.\mathrm{BAr}^{\mathrm{f}}\right), 131.1(p C \mathrm{H}$ arom IDiPP), 134.9 
(ipso Cq arom DiPP), 145.7 (o Cq arom DiPP), $162.2\left(\mathrm{q} 1: 1: 1: 1,{ }^{1} \mathrm{~J}_{\mathrm{CB}}=50 \mathrm{~Hz}, \mathrm{BAr}{ }^{\mathrm{F}}\right.$ ), 178.3 (s, NCN). IR (nujol): $2318 \mathrm{~cm}^{-1}\left(v\left(\mathrm{~N}_{-} \mathrm{C}\right)\right.$ ). Anal. Calcd for $\mathrm{C}_{64} \mathrm{H}_{56} \mathrm{BF}_{24} \mathrm{~N}_{3} \mathrm{Ni}$ : C, 55.20; H, 4.05; N, 3.02. Found: C, 55.22; H, 4.05; N, 3.12. MS (ESI) for $\mathrm{C}_{32} \mathrm{H}_{44} \mathrm{~N}_{3} \mathrm{Ni}^{+}$: $\mathrm{m} / \mathrm{z} 487.1\left(\mathrm{M}+-\mathrm{CH}_{3} \mathrm{CN}\right)$.

\section{Synthesis of $\left[\mathrm{Ni}\left(\eta^{3}-\mathrm{C}_{3} \mathrm{H}_{5}\right)(\mathrm{IMeMe})\left(\mathrm{NCCH}_{3}\right)\right] \mathrm{BAr}^{\prime}{ }_{4}(4 \mathrm{~b})$}

A solution of $\mathbf{2 b}(143 \mathrm{mg}, 0.2 \mathrm{mmol})$ in $10 \mathrm{~mL}$ of $\mathrm{CH}_{3} \mathrm{CN}$ at cooled $-40{ }^{\circ} \mathrm{C}$ was treated with a solution of $\mathrm{H}\left(\mathrm{Et}_{2} \mathrm{O}\right)_{2} \mathrm{BAr}_{4}{ }_{4}(280 \mathrm{mg}, 0.25 \mathrm{mmol})$ in $5 \mathrm{~mL}$ of $\mathrm{CH}_{3} \mathrm{CN}$. The mixture was stirred one hour at room temperature. The mixture was filtrated and the yellow residue of $\mathbf{4 b}$ washed with $20 \mathrm{~mL}$ of hexanes. Crystallization from toluene at $-30{ }^{\circ} \mathrm{C}$ afforded the product as yellow crystals in $50 \%$ yield. ${ }^{1} \mathrm{H} \mathrm{NMR}\left(\mathrm{CD}_{2} \mathrm{Cl}_{2}, 25^{\circ} \mathrm{C}\right): 2.04$ (partially obscured signal, $1 \mathrm{H}$, anti $\mathrm{CH} \mathrm{H}$ allyl), 2.07 (s, 6H, $\left.\mathrm{CH}_{3} \mathrm{C} \mathrm{IMeMe}\right), 2.20$ (s, 3H, $\mathrm{CH}_{3} \mathrm{CN}$ ), 2.88 (overlapping signal, $1 \mathrm{H}, \operatorname{syn} \mathrm{CH} \mathrm{H}$ allyl), 2.91 (overlapping signal, $1 \mathrm{H}$, ${ }^{3} J_{\mathrm{HH}} \approx 15 \mathrm{~Hz}$, anti $\mathrm{CHH}$ allyl), 3.71 (bs, $\left.6 \mathrm{H}, \mathrm{CH} \mathrm{H}_{3} \mathrm{~N} \mathrm{IMeMe}\right), 3.94\left(\mathrm{bd}, 1 \mathrm{H},{ }^{3} J_{\mathrm{HH}}=8 \mathrm{~Hz}\right.$, syn $\mathrm{CHH}$ allyl), $5.42\left(\mathrm{~m}, 1 \mathrm{H}\right.$, central $\mathrm{CH}$ allyl), $7.57\left(\mathrm{~s}, 4 \mathrm{H}, \mathrm{CH}\right.$ arom $\left.\mathrm{BAr}^{\mathrm{F}}\right), 7.73(\mathrm{~s}$, $8 \mathrm{H}, \mathrm{CH}$ arom BAr $\left.{ }^{\mathrm{F}}\right) .{ }^{13} \mathrm{C}\left\{{ }^{1} \mathrm{H}\right\} \mathrm{NMR}\left(\mathrm{CD}_{2} \mathrm{Cl}_{2}, 25^{\circ} \mathrm{C}\right): 3.7\left(\mathrm{CH}_{3} \mathrm{CN}\right), 8.8\left(\mathrm{~s}, \mathrm{CH}_{3} \mathrm{C}\right.$ IMe,Me), $34.8\left(\mathrm{CH}_{3} \mathrm{~N}\right), 50.3\left(\mathrm{CH}_{2}\right.$ allyl $), 70.5\left(\mathrm{CH}_{2}\right.$ allyl $), 113.9(\mathrm{CH}$ allyl $), 117.8(\mathrm{CH}$ arom $\left.\left.\mathrm{BAr}^{\mathrm{F}}\right), 121.3 \mathrm{q},{ }^{1} J_{\mathrm{CF}}=272 \mathrm{~Hz}, \mathrm{CF}_{3}, \mathrm{BAr}^{\mathrm{F}}\right), 127.3\left(\mathrm{CH}_{3} C, \mathrm{IMe}, \mathrm{Me}\right), 128.2(\mathrm{~s}$,

$\left.\mathrm{CH}_{3} C \mathrm{~N}\right), 129.2,\left(\mathrm{q},{ }^{2} J_{\mathrm{CF}}=31 \mathrm{~Hz}, C \mathrm{H}\right.$ arom $\left.\mathrm{BAr}{ }^{\mathrm{f}}\right), 162,1\left(\mathrm{q} 1: 1: 1: 1,{ }^{1} \mathrm{~J}_{\mathrm{CB}}=50 \mathrm{~Hz}\right.$, $\left.\operatorname{BAr}^{\mathrm{F}}\right), 171.0(\mathrm{~s}, \mathrm{NCN})$. IR (nujol): $2321 \mathrm{~cm}^{-1}\left(v\left(\mathrm{~N} \_\mathrm{C}\right)\right)$. Anal. Calcd for $\mathrm{C}_{44} \mathrm{H}_{32} \mathrm{BF}_{24} \mathrm{~N}_{3} \mathrm{Ni}: \mathrm{C}, 46.84 ; \mathrm{H}, 2.86 ; \mathrm{N}, 3.72$. Found: $\mathrm{C}, 46.50 ; \mathrm{H}, 2.53 ; \mathrm{N}, 3.57$.

\section{Butadiene polymerization.}

A $250 \mathrm{ml}$ glass reactor, with a magnetic stirring bar, and containing a $\mathrm{N}_{2}$ atmosphere was cooled in a bath of liquid nitrogen, and connected to a reservoir containing the desired amount of liquid butadiene $(10 \mathrm{ml})$. The stopcock was opened and butadiene was allowed to condense in the reactor. Next, $10 \mathrm{ml}$ of toluene were 
injected through a septum-capped port. The reactor was then closed and placed in a bath thermostatized at the working temperature $\left(30^{\circ} \mathrm{C}\right)$. A solution of the catalyst in toluene $(1 \mathrm{~mL})$ was injected through the septum port, and the mixture was stirred for $5 \mathrm{~h}$. Then, the volatile were evaporated under vaccuum, and collected in a liquid $\mathrm{N}_{2}$ trap. GC analysis of this liquid showed no evidence of light oligomers. The residue was dried under vacuum at $60^{\circ} \mathrm{C}$, weighed and analyzed by ${ }^{1} \mathrm{H},{ }^{13} \mathrm{C}$, and GPC.

\section{Styrene polymerization.}

Styrene $(10 \mathrm{ml}, 89 \mathrm{mmol})$, and toluene $(10 \mathrm{ml})$ were transferred to a glass ampoule, charged with a magnetic stirring bar and capped with a Young teflon valve, and taken to an oil bath maintained at $30^{\circ} \mathrm{C}$. Once the temperature of the mixture was judged to be stable, a solution of the catalyst in $1 \mathrm{ml}$ of toluene was injected through a septum, and the mixture stirred for $5 \mathrm{~h}$. After this time, a sample was removed for GC analysis. The solvent was stripped off and the residue dried under vacuum at $60{ }^{\circ} \mathrm{C}$. The

product obtained from catalyst 3 was a solid, whose ${ }^{1} \mathrm{H}$ and ${ }^{13} \mathrm{C}$ NMR spectra matched the expected for atactic polystyrene. Catalyst $\mathbf{4 b}$ produced a liquid product, whose NMR spectra and MS-GC chromatogram were consistent with a mixture of oligomers containing $85 \%$ of the head to tail dimer. 